\title{
CLASSICAL ANALOG TO THE AIRY WAVE PACKET
}

\author{
A. Matulis ${ }^{a}$ and A. Acus ${ }^{b}$ \\ a Semiconductor Physics Institute, Center for Physical Sciences and Technology, Sauletekio 3, 10222 Vilnius, Lithuania \\ ${ }^{\mathrm{b}}$ Institute of Theoretical Physics and Astronomy, Vilnius University, Sauletekio 3, 10222 Vilnius, Lithuania \\ Email: matulisalg@gmail.com; arturas.acus@tfai.vu.lt
}

Received 18 June 2019; accepted 25 June 2019

\begin{abstract}
The solution of the Liouville equation for the ensemble of free particles is presented and the classical analog to the quantum accelerating Airy wave packet is constructed and discussed. Considering the motion of various classical packets - with an infinite and restricted distribution of velocities of particles - and also the motion of their fronts, we demonstrate in the simplest and most definite way why the packet can display a more sophisticated behaviour (even acceleration) as compared with a free individual particle that moves at a fixed velocity. A comparison of this classical solution with the quantum one in the Wigner representation of quantum mechanics, which provides the closest analogy, is also presented.
\end{abstract}

Keywords: Liouville equation, accelerating front, density matrix in Wigner representation

\section{Introduction}

Due to dispersion in the Schrödinger equation, wave packets usually spread out during their propagation at a constant velocity in free space. However, almost half a century ago it was shown [1] that there existed a specific solution of that equation expressed in the Airy function which, on the one hand, did not change its form (that is why it is called a coherent state), and, on the other hand, it demonstrated an accelerating motion. These exotic solutions have recently aroused some interest [2] in possible applications towards the description of non-spreading optical beams [3] and the generation and control of plasma in dielectrics [4].

The abovementioned solution is also interesting from a didactic point of view because of an obvious contradiction between the quantum accelerating state and the classical free particle motion corresponding to it. Usually this contradiction is explained on the basis of the wave properties of the packet. In our view the main reason for that is a statistical nature of quantum mechanics. If this is the case, such strange packets should exist in clas- sical mechanics as well if particles were described statistically using the Liouville equation. The aim of this paper is to present solutions of that equation and to discuss the classical analog to the accelerating quantum coherent state corresponding to a free particle. To our mind, this classical problem is much simpler as compared to the quantum one, and consequently it may be helpful in understanding quantum mechanical problems better. In addition, we show that the analogy between the classical and quantum descriptions of the abovementioned sophisticated packets is quite close if the density matrix equation in the Wigner representation of quantum mechanics is used.

The paper is organized as follows. In Section 2 a description of the model is provided, convenient dimensionless variables are introduced, and the $\mathrm{Li}$ ouville equation for the ensemble of free particles is formulated. A general solution of that equation is given in Section 3. In the following three sections special cases such as an accelerating packet, a single particle and the packet of a restricted velocity distribution are discussed. Section 7 deals with the possible accelerating motion of the sharp front. 
In Section 8 classical solutions obtained are compared with the quantum ones given in the Wigner representation of quantum mechanics. Our conclusions are presented in Section 9.

\section{Model}

The quantum wave packet corresponding to the accelerating quantum particle is described by the following Schrödinger equation:

$$
\mathrm{i} \hbar \frac{\partial \Psi}{\partial t}=-\frac{\hbar^{2}}{2 m} \frac{\partial^{2} \Psi}{\partial x^{2}}-\max \Psi=0 .
$$

Here $m$ is the particle mass, $a$ is its acceleration, and $\hbar$ is the Planck constant. To make expressions more transparent we rescale time $t$, coordinate $x$ and velocity $v$ as follows:

$$
t \rightarrow t\left(\frac{\hbar}{m a^{2}}\right)^{1 / 3}, x \rightarrow x\left(\frac{\hbar^{2}}{m^{2} a}\right)^{1 / 3}, v \rightarrow v\left(\frac{\hbar a}{m}\right)^{1 / 3} .
$$

After this transformation the initial Eq. (1) can be presented in a simpler dimensionless form:

$$
\mathrm{i} \frac{\partial \Psi}{\partial t}=-\frac{1}{2} \frac{\partial^{2} \Psi}{\partial x^{2}}-x \Psi
$$

The dimensional expressions can be easily restored by applying the same transformation in the opposite direction.

As mentioned in the Introduction, Berry and Balazs have shown [1] that the Schrödinger equation for the free particle

$$
\mathrm{i} \frac{\partial \Psi}{\partial t}=-\frac{1}{2} \frac{\partial^{2} \Psi}{\partial x^{2}}
$$

has the solution

$$
\Psi(x, t)=\mathrm{e}^{\mathrm{i} t\left(x-t^{2} / 3\right)} \mathrm{Ai}\left[2^{1 / 3}\left(x-t^{2} / 2\right)\right],
$$

where the symbol Ai stands for the Airy function [5]. The probability corresponding to this wave function,

$$
P(x, t)=|\Psi(x, t)|^{2}=\mathrm{Ai}^{2}\left[2^{1 / 3}\left(x-t^{2} / 2\right)\right],
$$

is a function of the single argument $\left(x-t^{2} / 2\right)$ indicating that it is a coherent state related to the accelerating particle.
Now we are switching over to our main purpose, and we shall discuss the classical description of a similar motion. Instead of using Newton's equations for the individual particle we consider the ensemble of free particles and treat them statistically. The statistical description of classical particles is based on the Liouville theorem [6] which states that the distribution function $f(x, v, t)$ of the particles in the phase space (the density of particles in the $(x, v)$ plane in our case) behaves like an ideal liquid which remains constant when moving along the classical trajectories. This statement leads to the following Liouville equation

$$
\frac{\partial f}{\partial t}+v \frac{\partial f}{\partial x}=0
$$

for the distribution function of free particles. The solution of this equation enables us to express all properties of the particles via the integrals with the distribution function given above. For instance, the density of the particles as a function of coordinate and time is given as follows:

$$
n(x, t)=\int_{-\infty}^{\infty} \mathrm{d} v f(x, v, t) .
$$

This statistical technique is considered to be an analog to the solution of Newton's equations for individual particles.

\section{Solution of the Liouville equation}

The simplest way to find the solution of Eq. (7) corresponding to the accelerating motion is to look for a static solution in the accelerating frame $\{y, w\}$ which is related to the initial laboratory frame $\{x, v\}$ by the following equations:

$$
\begin{aligned}
& x=y+t^{2} / 2, \\
& v=w+t .
\end{aligned}
$$

In order to obtain the Liouville equation in the new frame it is necessary to make the following substitution of the derivatives in Eq. (7),

$$
\frac{\partial f}{\partial t} \rightarrow \frac{\partial f}{\partial t}-t \frac{\partial f}{\partial y}-\frac{\partial f}{\partial w}, \quad \frac{\partial f}{\partial x} \rightarrow \frac{\partial f}{\partial y},
$$

which leads to the following equation: 


$$
\frac{\partial f}{\partial t}+w \frac{\partial f}{\partial y}-\frac{\partial f}{\partial w}=0
$$

Actually it is the same Liouville equation including the additional force term that appears due to a non-inertial accelerating frame.

According to Courant [7], the first-order partial differential equation can be solved by means of the trajectory method. These trajectories (or characteristic curves) follow from the set of ordinary differential equations which are composed of Eq. (11) coefficients in the following way:

$$
\frac{\mathrm{d} t}{1}=\frac{\mathrm{d} y}{w}=\frac{\mathrm{d} w}{-1} .
$$

In solving these equations we obtain the trajectories

$$
\begin{aligned}
& y-w t-t^{2} / 2=y_{0}, \\
& w+t=w_{0}
\end{aligned}
$$

defined by two constants $y_{0}$ and $w_{0}$. Actually they are trajectories of individual particles if one describes them by means of Newton's equations. According to the abovementioned trajectory method, Eq. (11) means that the derivative of the distribution function along the trajectories (13) is equal to zero. Therefore the distribution function is constant along each of them, and the general solution of the Liouville equation (11) can be given as any function of those two constants characterizing the trajectory:

$$
f=f\left(y_{0}, w_{0}\right) \equiv f\left(y-w t-t^{2} / 2, w+t\right) .
$$

This expression will be used to obtain and discuss all specific solutions.

\section{Accelerating solution}

Let us start with the accelerating solution which is an analog to the quantum coherent state with the probability given by Eq. (6).

Eliminating time $t$ from Eqs. (13) we obtain the following equation,

$$
y+w^{2} / 2=y_{0}+w_{0} / 2 \equiv c_{0},
$$

which defines a set of fixed trajectories in the $(y, w)$ plane. They are shown in Fig. 1 by dashed parabo-

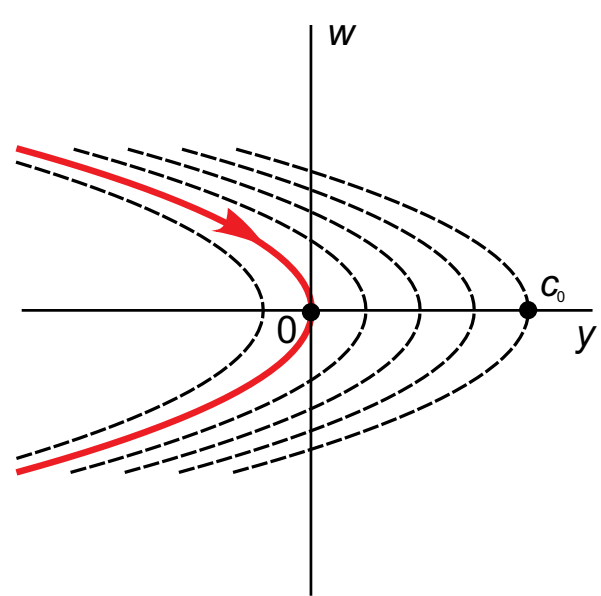

Fig. 1. The set of fixed trajectories in the $(y, w)$ plane of the accelerating frame.

las. The constant $c_{0}$ indicates the rightmost point of the parabola. One of them indicated by a (red online) solid curve corresponds to the $c_{0}=0$ value. Since the solution of the Liouville equation (14) can be chosen as any function of two constants $y_{0}$ and $w_{0}$, we choose it as a function of their expression given by Eq. (15),

$$
f=\delta\left(y_{0}+w_{0}^{2} / 2\right) \equiv \delta\left(y+w^{2} / 2\right),
$$

where the symbol $\delta(x)$ stands for the Dirac $\delta$-function. This function corresponds to the case, where all particles are located only on the red $\left(c_{0}=0\right)$ trajectory with constant density. This distribution can be regarded as a static one only nominally because from a microscopic point of view all particles move along the trajectories in the direction shown by the arrow.

Now by performing transformation (9) we obtain the following distribution function in the laboratory frame:

$$
f(x, v, t)=\delta\left(\left[x-t^{2} / 2\right]+[v-t]^{2} / 2\right) .
$$

As time $t$ appeared in the argument of the distribution function, the trajectories in the laboratory frame are no longer fixed. The motion of the trajectory corresponding to function (17) (to the red online trajectory shown in Fig. 1) is illustrated in Fig. 2, where time values are indicated by numbers on the curves. The rightmost points of the moving parabolas are shown by small solid (blue online) circles. They are located on the solid parabola

$$
x=v^{2} / 2,
$$




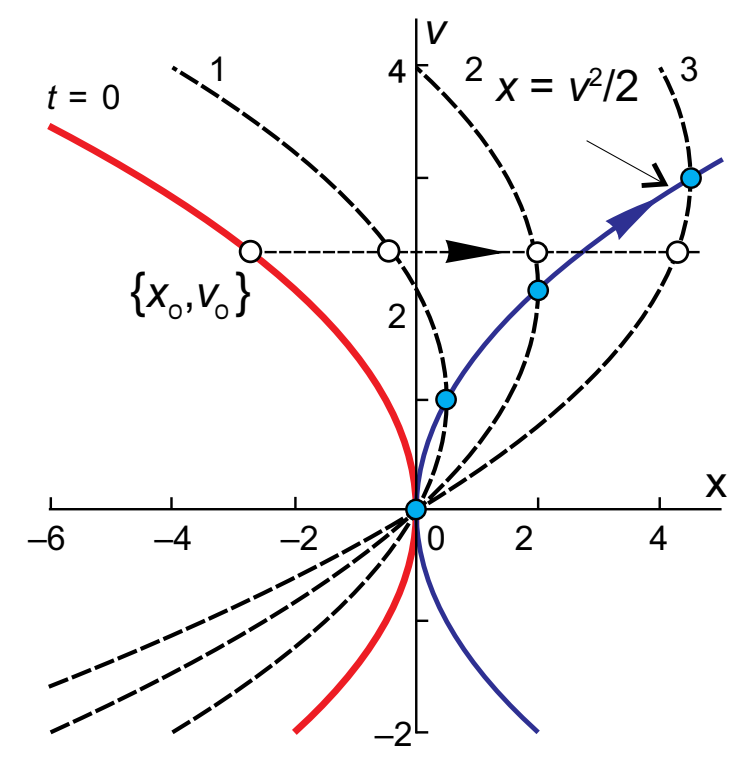

Fig. 2. Motion of the trajectory in the $(x, v)$ plane of the laboratory frame.

that is obtained by zeroing both brackets in the argument of function (17). This parabola corresponds to the accelerating particle with

$$
x=t^{2} / 2, v=t .
$$

This accelerating motion reveals itself more clearly when the density of particles is calculated by means of integral (8) with distribution function (17), namely,

$$
\begin{aligned}
& n(x, t)=\int_{-\infty}^{\infty} \mathrm{d} v \delta\left(\left[x-t^{2} / 2\right]+[v-t]^{2} / 2\right) \\
& =\frac{1}{\left|t-v_{+}\right|}+\frac{1}{\left|t-v_{-}\right|},
\end{aligned}
$$

where the zeroes of the $\delta$-function argument are as follows:

$$
v_{0 \pm}=t \pm \sqrt{t^{2}-2 x} .
$$

Consequently, the density of particles reads

$$
n(x, t)=\frac{2}{\sqrt{t^{2} / 2-x}} \Theta\left(t^{2} / 2-x\right),
$$

where the symbol

$$
\Theta(x)= \begin{cases}0, & x<0 \\ 1, & x \geq 0\end{cases}
$$

stands for the Heaviside step function.
We see that the density demonstrates radicaltype singularity that accelerates in the positive direction of the $x$ axis. It is shown in Fig. 3 by a thick solid (blue online) curve as a function of the $\left(x-t^{2} / 2\right)$ argument. For comparison the quantum mechanical result $(6)$ is indicated by a thin (red online) curve just below the classical one. Within the accuracy of normalization the classical result coincides with the envelope of the asymptote of the squared Airy function [5]

$$
|\operatorname{Ai}(-x)|^{2} \sim \frac{1}{\sqrt{\pi x}} \sin ^{2}\left(2 x^{3 / 2} / 3+\pi / 4\right),
$$

which confirms that the considered classical problem of free particles is an analog to the accelerating coherent quantum state. It leads us to the conclusion that the effect of acceleration is caused by the statistical properties of the classical system, as well as a statistical nature of the quantum system.

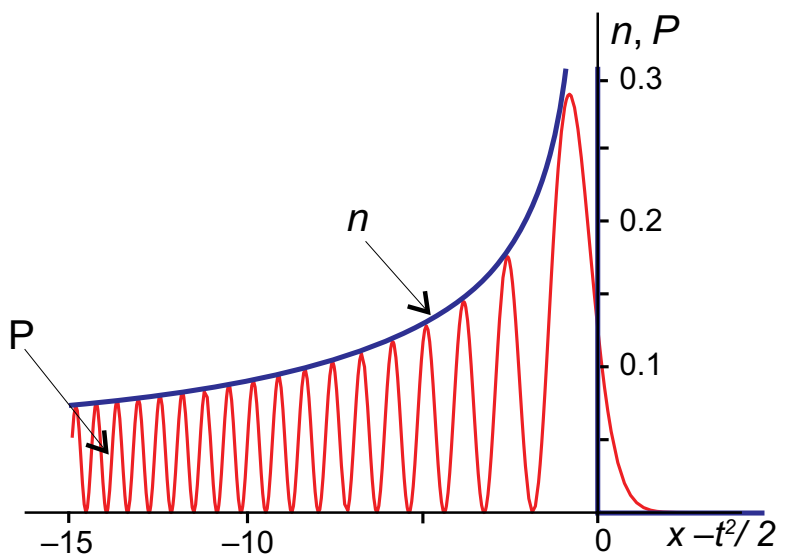

Fig. 3. Accelerating packet: a thin (red online) curve is the quantum mechanical result according to Eq. (6), a thick (blue online) curve is the classical result according to Eq. (22).

\section{Motion of an individual particle}

Now we shall show that the obtained accelerating state of the ensemble of particles does not contradict to the motion of a free individual particle which maintains its constant velocity.

The motion of an individual particle follows from the general solution of the Liouville equation (7) when the bellow-presented distribution function is chosen:

$$
f=\delta\left(y_{0}-x_{0}\right) \delta\left(w_{0}-v_{0}\right)
$$




$$
=\delta\left(y-w t-t^{2} / 2-x_{0}\right) \delta\left(w+t-v_{0}\right) .
$$

It corresponds to the particle which at the moment $t=0$ is located at the point $\left\{x_{0}, v_{0}\right\}$.

Using Eqs. (9) and going back to the laboratory frame we obtain the following distribution function,

$$
f(x, v, t)=\delta\left(x-v t-x_{0}\right) \delta\left(v-v_{0}\right),
$$

which shows that the individual particle moves along the trajectory

$$
v=v_{0}, \quad x=x_{0}+v_{0} t,
$$

maintaining its initial velocity $v_{0}$ indicated in Fig. 2 by a thin horizontal dotted line.

Consequently, the effect of acceleration is a pure statistical property of the ensemble of particles caused by a very specific initial condition when particles with a constant density are located on the infinite (red online) trajectory shown in Fig. 1 . This initial distribution includes particles of infinite velocities, which results in nonintegrable density.

\section{Motion of a packet}

The interesting question is whether it is possible to find an experimentally realizable initial condition when the packet demonstrates the accelerating motion. We shall try to answer this question modifying the distribution function (16), and adding some localization of velocities close to some average velocity $v_{0}$, namely choosing this function as follows:

$$
\begin{aligned}
& f(y, w)=\delta\left(c_{0}\right) \Phi\left(w_{0}-v_{0}\right) \\
& \equiv \delta\left(y+w^{2} / 2\right) \Phi\left(w+t-v_{0}\right) .
\end{aligned}
$$

Here the function $\Phi(v)$ stands for some symmetric local distribution of velocities. For instance, the Gaussian distribution

$$
\Phi(v)=\frac{1}{\sqrt{\pi D}} \mathrm{e}^{-v^{2} / D}
$$

may be chosen.

Going back to the laboratory frame we have

$$
f(x, v, t)=\delta\left(x+v^{2} / 2-v t\right) \Phi\left(v-v_{0}\right) .
$$

In a sense we can regard this distribution function as an intermediate solution between a nonphysical distribution (17) that demonstrates the accelerating motion and the distribution (26) corresponding to the individual particle that maintains a constant velocity. This distribution can be generated experimentally by injecting particles at the point $x=0$ with some retardation depending on the particle velocity. It might be expected that in changing the $D$ value it is possible to change gradually one type of motion to another one.

Let us check that possibility by calculating the mean coordinate of the packet,

$$
\begin{aligned}
& \langle x(t)\rangle=\int_{-\infty}^{\infty} \mathrm{d} v \int_{-\infty}^{\infty} \mathrm{d} x x f(x, v, t) \\
& =\int_{-\infty}^{\infty} \mathrm{d} v \Phi\left(v-v_{0}\right) \int_{-\infty}^{\infty} \mathrm{d} x x \delta\left(x+v^{2} / 2-v t\right) \\
& =\int_{-\infty}^{\infty} \mathrm{d} v \Phi\left(v-v_{0}\right)\left(v t-v^{2} / 2\right)=x_{0}+v_{0} t
\end{aligned}
$$

where

$$
x_{0}=-v_{0}^{2}-D / 4
$$

denotes the initial mean coordinate of the packet.

We see that the real packet of limited velocities demonstrates the motion at a fixed average velocity independently of the width $D / 4$ of the packet. This fact has been noticed [8] before when commenting on the motion of quantum mechanical packets composed of the superposition of the abovementioned Airy functions.

\section{The accelerating front}

Although we failed to construct the accelerating packet, we show that it is possible to construct the packet with some accelerating parts, for instance, with the accelerating front. In order to illustrate this possibility we consider the packet with a rectangle type distribution of velocities (the constant velocity in the region $0 \leq w \leq v_{0}$ ) on the initial (red online) trajectory shown in Fig. 1:

$$
\begin{aligned}
& f(y, w, t)=\delta\left(c_{0}\right) \Theta\left(w_{0}\right) \Theta\left(w_{0}-v_{0}\right) \\
& =\delta\left(y+w^{2} / 2\right) \Theta(w+t) \Theta\left(v_{0}-w-t\right) .
\end{aligned}
$$


Using Eqs. (9) we go back to the laboratory frame and obtain the following solution of the Liouville equation:

$$
f(x, v, t)=\delta\left(x+v^{2} / 2 a-v t\right) \Theta(v) \Theta\left(v_{0}-v\right) .
$$

The evolution of this trajectory is shown in Fig. A. Actually it is a horizontal stripe cut out of Fig. 2, which emerged owing to the restriction of velocities in the packet. Every point of the trajectory, say the one indicated by a (blue online) circle, moves to the right along a thin dotted horizontal line, and the initial (red online) trajectory moves to the right and upwards with its rightmost point $\left\{x_{k}, v_{k}\right\}$ moving along the (green online) parabola $x_{k}=v_{k}^{2} / 2$, as explained in Section 4 . The evolution of the coordinate and velocity corresponding to this point is shown in Fig. 5. We see that the acceleration of the front is evident. But it does not last forever. At the moment $t_{0}$ the rightmost point of the trajectory reaches the limiting velocity $v_{0}$, and its velocity no longer increases, as seen in Fig. 4. The reason for that is trivial: there are no more particles in the initial distribution with a larger velocity than $v_{0}$, and the front velocity stabilizes.

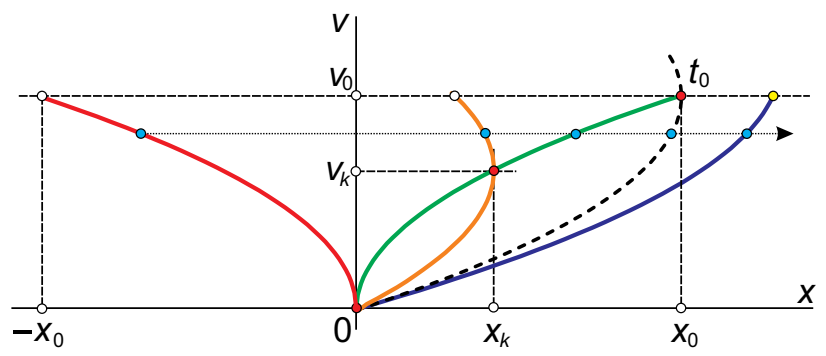

Fig. 4. Motion of the packet with a rectangle distribution of velocities (Eq. (33)).

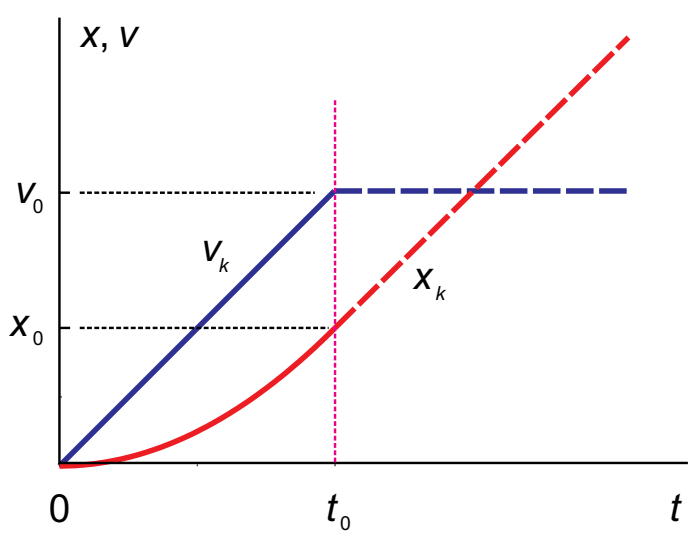

Fig. 5. Motion of the point $\left\{x_{k}, v_{k}\right\}$ corresponding to the right front of the packet.
In our opinion, this example clearly illustrates the difference between the motion of an individual particle described by an ordinary differential equation and the effects of the ensemble of particles described by a partial derivative equation. The latter one has many more degrees of freedom, and therefore it has many more various solutions, even some exotic ones. In the considered case the acceleration of the front takes place not only because of acceleration of individual particles but also because of their collective motion during which faster particles injected later overtake the slower ones, and consequently, the impression of the accelerating front forms. There are more effects of that type in physics. It is worth mentioning the difference in phase and group velocities of the wave in the dispersive media, or precursors [9] moving faster than the wave packets.

\section{Density matrix in the Wigner representation}

The analogy between the classical solution of the Liouville equation and the accelerating coherent quantum mechanical state that we demonstrated in the previous sections becomes even closer if the quantum state is described by a density matrix. It is known that the quantum mechanical description can be based not only on the wave function $\Psi(x, t)$ which satisfies the Schrödinger equation

$$
\mathrm{i} \frac{\partial}{\partial t} \Psi=\hat{H} \Psi
$$

but also on the density operator $\hat{\rho}$ that satisfies the quantum Liouville (or von Neumann) equation

$$
\mathrm{i} \frac{\partial}{\partial t} \hat{\rho}=[\hat{H}, \hat{\rho}] \text {. }
$$

When comparing Eqs. (4) and (35) we see that in our case of dimensionless variables the free particle Hamiltonian reads

$$
\hat{H}=-\frac{1}{2} \frac{\partial^{2}}{\partial x^{2}} \text {. }
$$

In the coordinate representation the density operator is usually presented by the density matrix which in the case of a pure state (in the case where the quantum system is characterized by the wave function) reads

$$
\hat{\rho} \rightarrow \rho\left(x_{1}, x_{2}, t\right)=\Psi\left(x_{1}, t\right) \Psi^{\star}\left(x_{2}, t\right) .
$$


In 1932 Wigner [10] proposed to replace this density matrix with the function

$$
F(x, v, t)=\int_{-\infty}^{\infty} \mathrm{d} \xi \mathrm{e}^{\mathrm{i} \xi v} \rho(x-\xi / 2, x+\xi / 2, t),
$$

which depends on the mean coordinate $x=\left(x_{1}+x_{2}\right) / 2$, and is the Fourier transform with respect to the relative coordinate $\xi=x_{2}-x_{1}$. All other operators of quantum mechanics have to be changed in the same way. It should be noted that we still use the same dimensionless variables. That is why there is no Planck constant in the above transformation, and the velocity $v$ coincides with the dimensionless momentum $p \rightarrow\left(\hbar m^{2} a\right)^{1 / 3} p$. The equations that are obtained in this way are called the Wigner representation of quantum mechanics [11]. Its main advantage is that in this representation quantum symbols and equations are quite similar to their classical counterparts. For instance, the coordinate and momentum operators convert themselves just to the numbers similar to the coordinate $x$ and the momentum $v$ used in classical mechanics. Their mean values are expressed in terms of simple integrals with the Wigner distribution function $F(x, v, t)$ :

$$
\begin{aligned}
& \langle x\rangle=\frac{1}{2 \pi} \int_{-\infty}^{\infty} \mathrm{d} x \int_{-\infty}^{\infty} \mathrm{d} v x F(x, v, t), \\
& \langle v\rangle=\frac{1}{2 \pi} \int_{-\infty}^{\infty} \mathrm{d} x \int_{-\infty}^{\infty} \mathrm{d} v v F(x, v, t) .
\end{aligned}
$$

The density of the particles is also given by the integral similar to its classical counterpart

$$
N(x, t)=\frac{1}{2 \pi} \int_{-\infty}^{\infty} \mathrm{d} v F(x, v, t) .
$$

Some price has to be paid for this simplicity and clearness: the product of two operators and their commutator have more complicated expressions. Hence, if in the Wigner representation the quantum mechanical operators $\hat{A}$ and $\hat{B}$ are replaced with the functions $a(x, v)$ and $b(x, v)$, respectively, their product and commutator have to be presented as follows:

$$
\begin{aligned}
& \hat{A} \cdot \hat{B} \rightleftarrows \exp \left[\frac{1}{2 \mathrm{i}}\left(\frac{\partial^{(a)}}{\partial v} \frac{\partial^{(b)}}{\partial x}-\frac{\partial^{(a)}}{\partial x} \frac{\partial^{(b)}}{\partial v}\right)\right] a b, \\
& {[\hat{A}, \hat{B}] \rightleftarrows \frac{2}{\mathrm{i}} \sin \left[\frac{1}{2}\left(\frac{\partial^{(a)}}{\partial v} \frac{\partial^{(b)}}{\partial x}-\frac{\partial^{(a)}}{\partial x} \frac{\partial^{(b)}}{\partial v}\right)\right] a b .}
\end{aligned}
$$

These equations are merely mnemonic rules presenting the Taylor expansions of the derivatives, where indexes show which function ( $a$ or $b$ ) they have to be applied to.

Although the sinus and exponent Taylor expansions are infinite, very often they break off when applied to polynomial-type functions. Such is the free particle Hamiltonian (37) which becomes a quadratic function of the momentum $H=v^{2} / 2$ in the Wigner representation. Due to this the sinus function in Eq. (42b) can be replaced with its argument, and the commutator can be rewritten as follows:

$$
\begin{aligned}
& {[H, \rho] \rightleftarrows \frac{2}{\mathrm{i}} \sin \left[\frac{1}{2}\left(\frac{\partial^{(v)}}{\partial v} \frac{\partial^{(F)}}{\partial x}-\frac{\partial^{(v)}}{\partial x} \frac{\partial^{(F)}}{\partial v}\right)\right] \frac{v^{2}}{2} F} \\
& =-\frac{\mathrm{i}}{2}\left(\frac{\partial^{(v)}}{\partial v} \frac{\partial^{(F)}}{\partial x}-\frac{\partial^{(v)}}{\partial x} \frac{\partial^{(F)}}{\partial v}\right) v^{2} F \\
& =-\frac{\mathrm{i}}{2} \frac{\partial^{(v)} v^{2}}{\partial v} \frac{\partial^{(F)} F}{\partial x}=-\mathrm{i} v \frac{\partial F}{\partial x} .
\end{aligned}
$$

Inserting this expression into Eq. (36) we finally obtain the following quantum Liouville equation in the Wigner representation

$$
\frac{\partial F}{\partial t}+v \frac{\partial F}{\partial x}=0
$$

which coincides exactly with the classical Eq. (7).

Consequently, in the case of simple systems (that of free particles) the density matrix in the Wigner representation satisfies the classical Liouville equation, and the quantum effects may reveal themselves only due to additional restrictions, such as the boundary or initial conditions, to be satisfied by the density matrix.

In order to understand better the difference between the classical and quantum solution of the Liouville equation we compute the Wigner function (39) in our case of the coherent state described by the wave function of Airy type (5),

$$
\begin{aligned}
& F(x, v, t)=\int_{-\infty}^{\infty} \mathrm{d} \xi \mathrm{e}^{\mathrm{i} \xi(v-t)} \mathrm{Ai}\left[2^{1 / 3}(X-\xi / 2)\right] \\
& \times \operatorname{Ai}\left[2^{1 / 3}(X+\xi / 2)\right],
\end{aligned}
$$

where

$$
X=x-t^{2} / 2 \text {. }
$$


This integral can be calculated analytically taking advantage of the integral representation of the Airy function

$$
\operatorname{Ai}(x)=\frac{1}{2 \pi} \int_{-\infty+\mathrm{i} \alpha}^{\infty+\mathrm{i} \alpha} \mathrm{d} u \mathrm{e}^{\mathrm{i}\left(x u+u^{3} / 3\right)},
$$

where the contour of integration is shifted up in the complex $u$ plane by small quantity a to insure convergence of the integral. Now denoting $P=v-t$, $a=2^{1 / 3}$ and omitting the integral limits (they are the same as in integral (47)), we write down the Wigner function as follows:

$$
\begin{aligned}
& F=\frac{1}{(2 \pi)^{2}} \int_{-\infty}^{\infty} \mathrm{d} \xi \mathrm{e}^{\mathrm{i}(P-a u / 2+a s / 2) \xi} \\
& \times \int \mathrm{d} u \mathrm{e}^{\mathrm{i} u^{3} / 3+\mathrm{i} a X u} \int \mathrm{d} s \mathrm{e}^{\mathrm{i} s^{3} / 3+\mathrm{i} a X s} \\
& =\frac{1}{2 \pi} \int \mathrm{d} u \mathrm{e}^{\mathrm{i} u^{3} / 3+\mathrm{i} a X u} \int \mathrm{d} s \mathrm{e}^{\mathrm{i} s^{3} / 3+\mathrm{i} a X s} \\
& \times \delta(P-a u / 2+a s / 2) \\
& =\frac{1}{\pi a} \int \mathrm{d} u \mathrm{e}^{\mathrm{i} u^{3} / 3+\mathrm{i} a X u} \mathrm{e}^{\mathrm{i}(u-P / a)^{3} / 3+\mathrm{i} a X(u-P / a)} .
\end{aligned}
$$

Finally substituting $u=(\eta+P) / a$ we rewrite this integral as

$$
F=\frac{a}{2 \pi} \int \mathrm{d} \eta \mathrm{e}^{\mathrm{i}\left[\eta^{3} / 3+2 \eta\left(P^{2} / 2+X\right)\right]},
$$

which according to Eq. (47) lets one to express this Wigner function in terms of the Airy function:

$$
F(x, v, t)=2^{1 / 3} \operatorname{Ai}\left(2\left[(v-t)^{2} / 2+\left(x-t^{2} / 2\right)\right]\right) .
$$

We see that the argument of this function coincides with the argument of the classical distribution function (17) which proves that the Wigner function of the considered pure coherent Airy state can be constructed in the same way as the classical solution of the Liouville equation, namely, as some constant distribution along the classical trajectories. The only difference is that in the quantum case the constants characterizing different trajectories cannot be chosen arbitrary which indicates a well-known fact that the quantum solution is stronger correlated as compared with the classical one. This correlation appears as oscillations, the characteristic period of which, according to Eq. (2), is proportional to $\hbar^{2 / 3}$. Inciden- tally, this result illustrates a plain fact that the limiting transition of quantum mechanics to classical one at $\hbar \rightarrow 0$ is quite complicated: the quantum oscillations disappear due to an increase in their frequency rather than due to a decrease in their amplitude.

Meanwhile the density calculated by the integral (41) with the Wigner function (50) coincides with the one obtained by Eq. (6) that we have checked numerically.

\section{Conclusions}

We presented a general solution of the Liouville equation describing an ensemble of free particles. In considering various specific cases we showed that although individual particles moved at a fixed velocity, the packet constructed of them can demonstrate quite a different behaviour. If the packet includes the particles with unrestricted velocities, it can even accelerate, and thus, it can become an analog to the quantum coherent accelerating state described by the Airy function.

The mean coordinate of the packet which is composed of the particles with restricted velocities does not show any acceleration. However, even in this case it is possible either to choose the initial condition or inject particles in a correlated way so that some parts of the packet, say, its front, should accelerate during the finite time interval. The essence of this motion is that the particles which are injected later overtake the slowest ones, and in this way create the image of the accelerating front.

These conclusions are applied in the case of the quantum free particle because the density matrix equation in the Wigner representation for this simple system coincides with the classical Liouville equation.

\section{Acknowledgements}

This research was partly (A. Acus) funded by the European Social Fund under Grant No. 09.3.3-LMTK-712-01-0051. The authors are sincerely grateful to Professor Kęstutis Pyragas for a detailed discussion of the work and his helpful comments.

\section{References}

[1] M.V. Berry and N.L. Balazs, Nonspreading wave packets, Am. J. Phys. 47(3), 264-267 (1979). 
[2] V.M. Vyas, Airy wavepackets are Perelomov coherent states, Am. J. Phys. 86(10), 750-754 (2018).

[3] G.A. Siviloglou and D.N. Christodoulides, Accelerating finite energy Airy beams, Opt. Lett. 32(8), 979-981 (2007).

[4] P. Polynkin, M. Kolesnik, J.V. Moloney, G.A. Siviloglou, and D.N. Christodoulides, Curved plasma channel generation using ultraintense Airy beams, Science 324(5924), 229-232 (2009).

[5] M. Abramowitz and I.A. Stegun, Handbook of Mathematical Functions, Sec. 10.4 (National Bureau of Standards, Washington, 1972).

[6] H. Goldstein, C. Poole, and J. Safko, Classical Mechanics (Addison Wesley, New York, 2000), p. 419.
[7] R. Courant and D. Hilbert, Methods of Mathematical Physics, Vol. 2 (John Wiley \& Sons, New York, 1989), 5, p. 28.

[8] J. Lekner, Airy wavepacket solutions of the Schrödinger equation, Eur. J. Phys. 30, L43-L46 (2009).

[9] A. Matulis, M. Zarenia, and F.M. Peeters, Wave fronts and packets in 1D models of different meta-materials: Graphene, left-handed media and transmission line, Phys. Status Solidi 10, 2330-2338 (2015).

[10]E. Wigner, On the quantum correction for thermodynamic equilibrium, Phys. Rev. 40, 749-759 (1932).

[11]S.R. Groot and L.G. Suttorp, Foundations of Electrodynamics, Ch. 6 (North-Holland Publishing Company, Amsterdam, 1971).

\title{
KLASIKINIS BANGINIO AIRY PAKETO ANALOGAS
}

\author{
A. Matulis ${ }^{a}$, A. Acus ${ }^{b}$ \\ ${ }^{a}$ Fiziniu ir technologijų mokslų centro Puslaidininkiu fizikos institutas, Vilnius, Lietuva \\ ${ }^{\mathrm{b}}$ Vilniaus universiteto Teorines fizikos ir astronomijos institutas, Vilnius, Lietuva
}

\section{Santrauka}

Pateiktas Liuvilio lygties sprendinys, aprašantis laisvų dalelių ansambli, ir sukonstruotas bei aptartas klasikinis kvantinio greitejančio banginio Airy paketo analogas.

Sugretinus ịvairius klasikinius begalinio ir riboto daleliu greičių pasiskirstymų paketus, pačiu paprasčiausiu būdu parodyta, kodèl iš laisvujų dalelių, judančių pastoviu greičiu, sudarytas paketas gali elgtis gana ịvairiai (taip pat ir greitèti). Šie klasikinio paketo judèjimo ypatumai palyginti su kvantinę dalelę aprašančiais tankio matricos lygties sprendiniais Vignerio vaizdavime, kur klasikinio bei kvantinio aprašymų analogija yra ryškiausia. 